\title{
Blood Flow and Velocity Estimation Based on Vessel Transit Time by Combining 2D and 3D X-Ray Angiography
}

\author{
Hrvoje Bogunović and Sven Lončarić \\ Faculty of Electrical Engineering and Computing \\ University of Zagreb, Unska 3, HR-10000 Zagreb, Croatia \\ $\{$ hrvoje.bogunovic, sven.loncaric\}@fer.hr \\ http://ipg.zesoi.fer.hr
}

\begin{abstract}
The X-ray imaging equipment could be used to measure hemodynamic function in addition to visualizing the morphology. The parameters of specific interest are arterial blood flow and velocity. Current monoplane X-ray systems can perform 3D reconstruction of the arterial tree as well as to capture the propagation of the injected contrast agent on a sequence of $2 \mathrm{D}$ angiograms. We combine the $2 \mathrm{D}$ digital subtraction angiography sequence with the mechanically registered 3D volume of the vessel tree. From 3D vessel tree we extract each vessel and obtain its centerline and cross-section area. We get our velocity estimation from $2 \mathrm{D}$ sequence by comparing time-density signals measured at different ends of the projected vessel. From the average velocity and cross-section area we get the average blood flow estimate for each vessel. The algorithm described here is applied to datasets from real neuroradiological studies.
\end{abstract}

\section{Introduction}

Medical imaging in addition to diagnostic purpose, is also being used as an interventional and therapeutic tool in order to perform minimally invasive treatment of vascular diseases. Since these interventions are altering the hemodynamic function, the physiologic parameters of interest are blood flow and velocity in arteries.

X-ray imaging is still considered as a gold standard for endovascular procedures so it would be of great interest if the same imaging device could be used to immediately evaluate the result of the intervention. Additionally, X-ray equipment is readily available in both developed and developing countries. It can be used to image $3 \mathrm{D}$ volumes and it is very suitable for taking a sequence of dynamic images because high frame rate does not deteriorate the spatial image resolution as much as in the other imaging modalities. However some difficulties arise due to its projective imaging and the limitation of the dose patient is allowed to receive.

There are already a number of publications that tackle the problem of blood flow and velocity estimation with X-ray systems. A thorough review of algorithms was given in [1. However, few of them had access to the $3 \mathrm{D}$ volume of 
vessels that are being imaged by digital subtraction angiography (DSA) since $3 \mathrm{D}$ reconstruction angiography (3DRA) was not largely available at that time.

We will focus on the techniques which are based on tracking of the intra arterially injected iodinated contrast agent. We found other approaches to either demand a rarely available contrast injection procedure or proved to be computationally too intensive.

The algorithms for blood velocity estimation based on tracking can in general be divided into the following categories [2]:

- Techniques based on measuring the transit time of contrast medium from one region of the vessel to the other 1 .

- Techniques based on measuring the distance that contrast medium travels between two frames 32 .

- Optical flow based methods 45/2].

In essence, all of them rely on signals which are obtained by observing the image intensity (density) level which changes through time (time-density signal) and/or space (distance-density signal) as the contrast agent passes through the vascular system.

Techniques based on measuring the distance the contrast medium travels between two frames by comparing distance-density signals in them are regarded as more accurate than time-density based methods. This is because the spatial resolution is normally higher than temporal one and the obtained velocity is localized in time and space. Time-density methods are capable of giving only the average velocities and are difficult to interpret when pulsatile flow is in effect. Optical flow-based methods are using 1D optical flow constraint (contrast agent is preserving its shape between two frames) for efficient distance-density curve matching which is otherwise done by exhaustive search. This matching is accurate when the gradient of the signal is strong which is only during the traversal of the leading or the trailing edge of the contrast agent.

All of these methods require imaging with high number of frames per second (at least 25). Otherwise, the contrast agent either moves to much between two frames or time-density signals in two distinct regions are too close to each other.

Once the blood velocity $(v)$ is known, the blood flow $(Q)$ is obtained by multiplication of this velocity with vessel cross-section area $(d A)$. In general, flow is a dot product of the velocity vector and the normal of area element.

$$
Q=\iint \boldsymbol{v} \cdot d \boldsymbol{A}
$$

In the case the flow is perpendicular to area and has a uniform velocity distribution across it (assumption of plug flow), we get a simple form.

$$
Q=v A
$$

Most of the current methods were validated on phantoms or computer simulations. We wanted to develop an algorithm, for use with monoplane X-ray 
imaging, that would be especially appropriate for realistic clinical settings with live patients. However validation of in-vivo studies proved to be difficult.

We focus on the application in the neuroradiology where pulsation in the flow should not be that strong as in the regions closer to the heart. In order to have a valid registration between $3 \mathrm{D}$ and $2 \mathrm{D}$ datasets, patient should not move between the two acquisitions. It is relatively easy to fixate the patient's head to prevent the movement. Neuro cases are of very high interest due to large number of vascular diseases like aneurysms, stenosis, and arteriovenous malformations that occur there and threaten to cause a stroke. This leads to a large amount of endovascular treatments which are performed there.

\section{Proposed Algorithm}

Our approach is based on registering the $3 \mathrm{D}$ vessel tree and $2 \mathrm{D}$ dynamic DSA sequence thus enabling comparison of time-density (TD) signals in the two opposite ends of the vessel. We think the TD based method is more robust than using distance-density (DD) oriented algorithm. For DD signals to be useful, the distance the contrast agent passes has to be reconstructed in $3 \mathrm{D}$ because we observe only the $2 \mathrm{D}$ projection, thus correction for angulation between imaged vessel and emitted X-rays has to be performed. Secondly, projected vessels can overlap and the parts of the vessel covered by other vessels have to be neglected and distance-density signal adequately interpolated, which is a difficult task.

Both the TD and DD signals are heavily cluttered with noise so special attention has to be given to adequate denoising procedures. In the previous research we focused on denoising techniques of these signals based on linear Wiener filtering and non-linear denoising using wavelet transform [6]. The 1D signals that we will show later in the paper will be already filtered.

The algorithm for automatic estimation of the blood velocity and flow, using 3D volume of arteries and sequence of 2D DSA images, that we used and performed tests with, can be summarized into these steps:

1. Vessel tree is first segmented out from the raw volume data.

2. Every vessel of the vessel tree is detected and labeled. Vessels are segments of the tree with no bifurcations. For each of these vessels, we are interested in its blood flow value. During this process the 3D centerline together with distance transform of vessels is computed. From the distance transform, cross-section area of the vessel is found. Centerline is also required to find the actual length of the vessel in $3 \mathrm{D}$.

3. Each extracted $3 \mathrm{D}$ vessel is projected onto 2D image thus automatically segmenting this vessel in $2 \mathrm{D}$ image sequence. This would otherwise be very difficult task to do by just observing $2 \mathrm{D}$ images. Also overlap with other vessels is detected.

4. Contrast propagation in each vessel is recovered from $2 \mathrm{D}$ sequence. Vessel transit time is estimated which together with known actual vessel length gives us the average blood velocity in the vessel.

5. Finally, blood flow for each vessel is found using Eq. 2. 


\subsection{Vessel Tree Volume}

Monoplane X-ray imaging equipment is capable of making 3D reconstruction of the imaged volume from a series of images taken at different angles. From this volume, vessel tree segmentation has to be performed. Simple global thresholding proved to be sufficient for our datasets.

Skeletonization of the segmented 3D binary object is performed based on the algorithm described in 7 . The result contains centerlines for each vessel of the tree. Additionally since the skeletonization procedure is based on distance transform every centerline voxel has its distance to nearest boundary assigned. Now labeling of the original segmented vessel tree can be performed. The result is shown in Fig. 1 (a), Every vessel is assigned a different label (shade of gray).

From the labeled vessel tree, each vessel of interest can be extracted. Since we based our method on comparing two TD signals, we first need to define two segments of the vessel that are on the opposite ends in which we will measure the signals. So we have to partition the vessel into smaller segments. To create vessel segments, we first create segment's border planes which are perpendicular to the centerline and the distance between these planes is chosen to be around ten voxels. Segment's volume within these borders is then obtained by region growing. Result of such partitioning is shown in Fig. 1 (b)

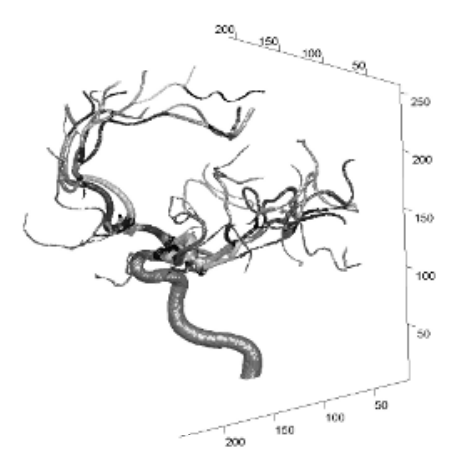

(a)

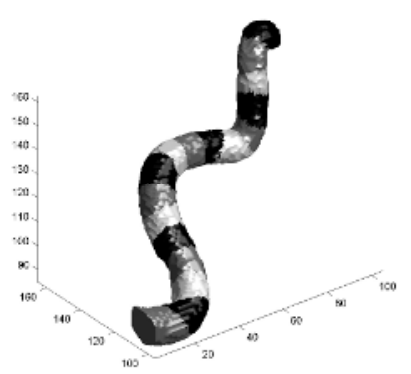

(b)

Fig. 1. Vessel tree labeling (a) and vessel partitioning (b)

\subsection{Projection of 3D Volume onto 2D Plane}

Once we obtain the necessary vessel data in 3D, we need to make a link with the $2 \mathrm{D}$ sequence showing contrast propagation. Since the imaging setting is known we can project accurately the $3 \mathrm{D}$ volume onto the $2 \mathrm{D}$ imaging plane. Thus the $3 \mathrm{D}-2 \mathrm{D}$ registration is mechanically based. The projection is perspective and is performed by ray tracing. The imaging setting and the corresponding projection of the vessel tree onto the 2D image are shown in Fig. 2. 


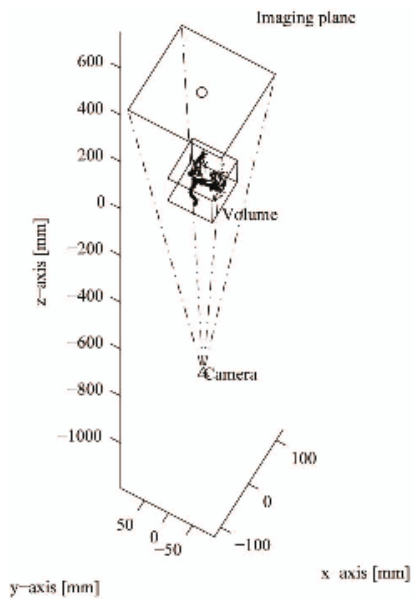

(a) Imaging setting

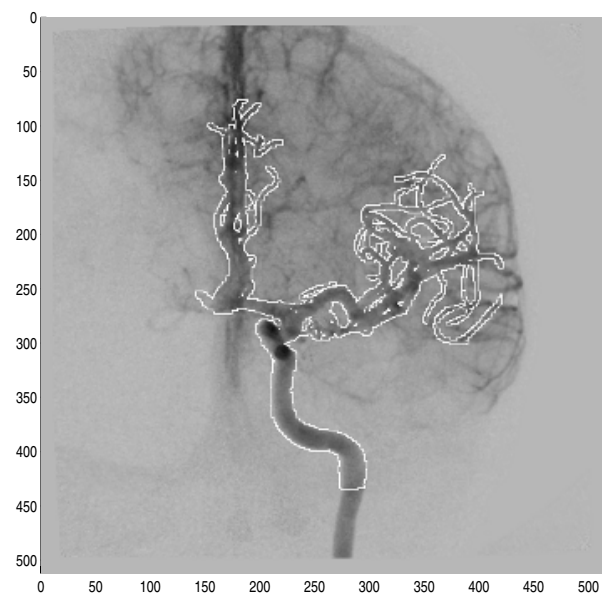

(b) Projected vessel tree (in white)

Fig. 2. Projection of the 3D vessel tree

Together with the vessel we project its two segments of interest in which we measure TD signal. Since ray tracing is used, we can easily detect and avoid segments with vessel overlap and choose the two most distant ones which are overlap-free. Such two projected overlap-free segments in vessel which generally overlaps with other vessels are shown in Fig. 3. Vessel of interest is shown in dark gray while the two segments chosen are shown in light gray.

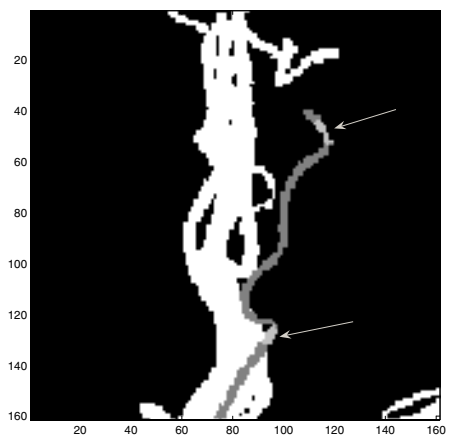

Fig. 3. Projection of two valid segments

\subsection{Vessel Transit Time}

When we have two regions of interest on the imaging plane we can measure the TD signal in them. From TD signals we need to estimate time of arrival (TA) of contrast agent to each of the two chosen regions. Vessel transit time (VTT) is obtained as a difference between these two TA values. TA can in general be found using two different criteria: peak to peak or half-peak to half-peak method. 
Contrast agent during its travel generates TD signal with three distinct phases: wash-in, maximum opacification and wash-out. Normally, TA would be taken as the peak of the signal during the maximum opacification phase which is the least affected by dilution. However, the problem is with signals which have steady maximum opacification yielding flat profile with unknown exact peak value. To overcome this problem, the moment the TD signal reaches its half-peak value can be taken as TA which normally occurs during the wash-in phase where the signal is quite steep. The nice property of this approach is that all values close to the flat peak will have very similar TA parameter (Fig. 4). Subframe accuracy can be achieved with suitable interpolation.

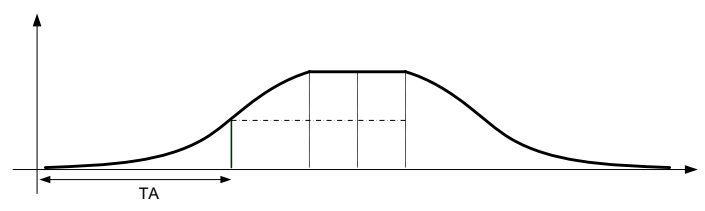

Fig. 4. Half-peak method for VTT estimation

From TD signals, one actually detects the frame number of the sequence when the contrast arrives. The difference in frame numbers $(\Delta N)$ is transformed into seconds using frames per second (FPS) parameter of the imaging study.

In addition to VTT, from the $3 \mathrm{D}$ centerline we know the distance between the two segments as well as the average radius of the vessel so we can find both the average blood velocity and the flow in the vessel.

\section{Results}

In this section, experimental results of estimating average blood velocity and flow will be presented. The above algorithm is performed automatically on every vessel of the vessel tree. Here we will show the results for the internal carotid artery (ICA) vessel.

Result of carotid vessel partitioning was already shown in Fig. 1, (b), Result of the projection is shown in Fig. 5. It is seen (light gray) how couple of segments from the top side had to be skipped because vessel is overlapping with itself (centerline is parallel with X-rays). The more lighter gray part of the chosen segments is the one actually used for TD signal construction since the rest of the segment had to be discarded due to the overlap with neighbouring segments.

TD signals are measured in the two segments from DSA sequence as average intensity value per frame of all the pixels in the segment. We estimate both the peak to peak distance as well as half-peak to half-peak distance. This is shown in Fig. 6. Since it is difficult to get images with high frames-per-second imaging setting, the arrival times to the two regions of the vessel are very close to each other. The final estimated values for this example are shown in Table 1. For this 


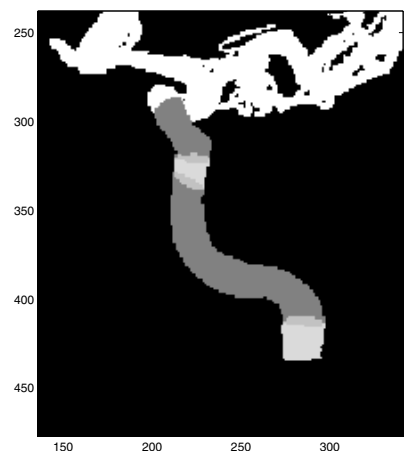

Fig. 5. Projected vessel tree with its segments (close up)

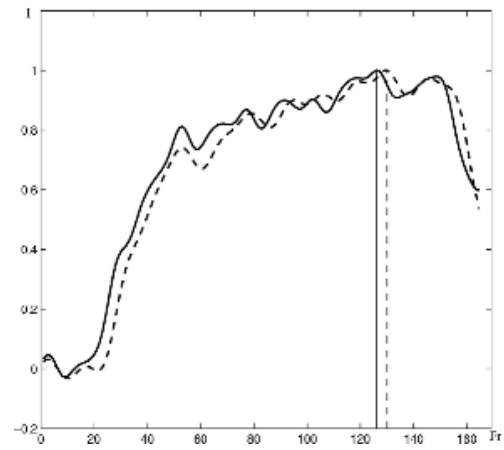

(a) peak to peak method

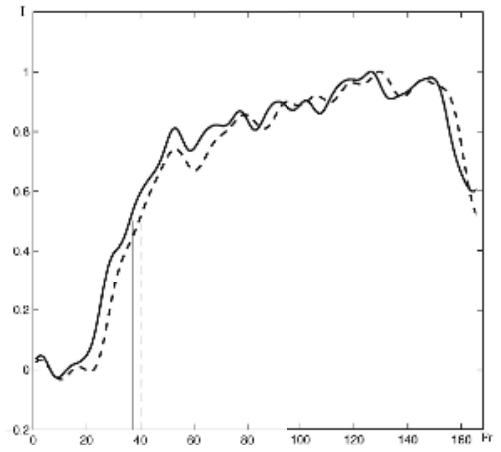

(b) half-peak to half-peak method

Fig. 6. VTT estimation (Normalized intensity vs. Frame number)

Table 1. Results for carotid artery

\begin{tabular}{|l|c|c|}
\hline parameter & peak-peak & half peak-half peak \\
\hline \hline$\Delta S[\mathrm{~mm}]$ & 40 & 40 \\
$\Delta N[$ frames $]$ & 4 & 3.3 \\
$\Delta T[\mathrm{~s}]$ & 0.16 & 0.13 \\
Diameter $[\mathrm{mm}]$ & 5.8 & 5.8 \\
\hline$v[\mathrm{~cm} / \mathrm{s}]$ & 25 & 30.7 \\
$Q[\mathrm{ml} / \mathrm{min}]$ & 392 & 481.5 \\
\hline
\end{tabular}

case both methods give similar result for VTT since the signal around its peak does not become flat as in some other, smaller vessels.

For the validation, we can only compare our values with the typical ones that appear in ICA, usually obtained by Doppler ultrasound. Although it is difficult to say what are normal velocities in ICA, we have found reports that the mean peak systolic value (PSV) should be in the range from 30 to $80 \mathrm{~cm} / \mathrm{sec}$, and is 
not viewed as abnormal until PSV exceeds $100 \mathrm{~cm} / \mathrm{sec}$. Although the valid range is quite large, it at least proves that our obtained results have meaningful values.

\section{Conclusion}

In this paper we discuss a technique for estimating blood velocity and flow in the arteries of the brain using monoplane X-ray imaging system. Estimation had to be supported with the mechanically registered 3D volume of the vessel tree obtained with the same device just before the DSA study. Velocity calculation is based on estimating vessel transit time from TD signals in two distant regions of the vessel. The results are in the expected range of values commonly found in the specific vessels.

In the future work, we plan to compensate for the vessel overlapping by suitable interpolation, and do appropriate correction for angulation of the vessels. This will enable us to focus more on the development of DD based methods which have a number of previously discussed advantages over TD based ones.

\section{References}

1. Shpilfoygel, S.D., Close, R.A., Valentino, D.J., Duckwiler, G.R.: X-ray videodensitometric methods for blood flow and velocity measurement: A critical review of literature. Medical Physics 27 (2000) 2008-2023

2. Rhode, K.S., Lambrou, T., Hawkes, D.J., Seifalian, A.M.: Novel approaches to the measurement of arterial blood flow from dynamic digial x-ray images. IEEE Trans. on Medical Imaging 24 (2005) 500-513

3. Shpilfoygel, S.D., Close, R.A., Valentino, D.J., Duckwiler, G.R.: Comparison of methods for instantaneous angiographic blood flow measurement. Medical Physics 26 (1999) 862-871

4. Huang, S.P., Decker, R.J., Goodrich, K.C., Parker, D.J., Muhlestein, J.B., Blatter, D.D., Parker, D.L.: Velocity measurement based on bolus tracking with the aid of three-dimensional reconstruction from digital subtraction angiography. Medical Physics 25 (1997) 677-686

5. Rhode, K.S., Lambrou, T., Hawkes, D.J., Hamilton, G., Seifalian, A.M.: Validation of an optical flow algorithm to measure blood flow waveforms in arteries using dynamic digital x-ray images. In: SPIE Medical Imaging: Image Processing. (2000) 1414-1425

6. Bogunovic, H., Loncaric, S.: Denoising of time-density data in digital subtraction angiography. In: Scandinavian Conference on Image Analysis. (2005) 1157-1166

7. Farag, A.A., Hassouna, M.S., Falik, R., Hushek, S.: Reliable fly-throughs of vascular trees. Technical report, CVIP Laboratory, University of Louisville (2005) 